\title{
Psychosocial impact of COVID-19 pandemic lockdown on people living with eye diseases in the UK
}

\author{
Darren Shu Jeng Ting $\mathbb{D}^{1} \cdot$ Sherine Krause ${ }^{2} \cdot$ Dalia G. Said ${ }^{1} \cdot$ Harminder S. Dua ${ }^{1}$
}

Received: 8 July 2020 / Revised: 30 July 2020 / Accepted: 3 August 2020 / Published online: 10 August 2020

(c) The Royal College of Ophthalmologists 2020

\section{To the Editor:}

In response to the Coronavirus disease 2019 (or COVID-19) pandemic, many countries across the world, including the UK, had imposed nationwide lockdown. Ophthalmology represents one of the busiest outpatient-based specialties, and cancellation of routine services meant that thousands of eye patients had been affected. Several studies have previously shown that COVID-19 lockdown and quarantine in general could negatively impact on the mental health of adults, children, and healthcare workers [1-3], but the impact of people with eye diseases has not been evaluated. In this survey, we aimed to evaluate the psychosocial impact of COVID-19 lockdown on people living with eye diseases in the UK.

In May 2020, a 27-item online questionnaire-based survey was administered to individuals ( $\geq 18$ years old) with eye diseases (Supplementary Table 1). Participants were identified through various eye support charities or support groups. The level of visual impairment (VI) was categorised into normal/mild, moderate, and severely sight impaired/ blind. Statistical analysis was performed using IBM SPSS Statistics v26. Multivariable logistic regression analysis was conducted to determine the predictive factors for worsened mental health, loneliness, and fear of further sight loss

Supplementary information The online version of this article (https:// doi.org/10.1038/s41433-020-01130-4) contains supplementary material, which is available to authorized users.

\footnotetext{
$\triangle$ Darren Shu Jeng Ting

ting.darren@gmail.com

$\triangle$ Harminder S. Dua

profdua@gmail.com

1 Academic Ophthalmology, Division of Clinical Neuroscience, School of Medicine, University of Nottingham, Nottingham NG7 2RD, UK

2 Fight for Sight, London, UK
}

during lockdown. Ethical approval of this survey was waived as no medical assessment/intervention was performed. Informed consent for publication was obtained from all participants.

Of all 325 respondents, $64.3 \%$ were $\geq 65$ years and $71.1 \%$ were female (Supplementary Table 2). The level of VI was none/mild (34.8\%), moderate (38.2\%), severe/blind (24.9\%), and unmentioned $(8.3 \%)$. The lockdown negatively impacted on the mental health $(45.9 \%)$, loneliness $(46.0 \%)$, social life $(85.3 \%)$, anxiety related to hospital visits $(55.1 \%)$, and fear of further sight loss due to delayed review/treatment (45.9\%), amongst others (Fig. 1). In addition, 39.2\% respondents mentioned that their eye diseases had made it more difficult to cope with the lockdown. Logistic regression analyses demonstrated that the lockdown had significantly affected the mental health of people who were of 18-64 years (OR 3.16; $p<0.001)$, and with moderate (OR $2.61 ; p=0.001)$ or severe VI (OR 2.07; $p=0.044$; Table 1$)$. People with moderate or severe VI were three times more likely to experience increased loneliness than those with mild/no VI $(p<0.001$ and $p=$ 0.002 , respectively). Fear of further sight loss was similar across all age groups, gender, and extent of VI (all $p>0.05$ ). The open comments reflected anxiety, stress, and fear impacting on mental health, compounded by the limitations posed by their VI on undertaking routine activities or providing care to other family members.

Sight loss is a big health concern, and delay in follow-up and care could result in significant and/or permanent harm to vision [4]. We highlighted that COVID-19 lockdown could pose a significant negative psychosocial impact on people with VI. It is therefore important to recognise and provide additional psychosocial support to this vulnerable group of people during the pandemic [3].

COVID-19 pandemic has posed challenges but also presented new opportunities. In view of the significant backlog of work and ongoing social distancing measures, adoption of telemedicine in ophthalmology will likely become the new "norm" of ophthalmology during and after the pandemic [5]. 


\section{Psychosocial impact of COVID-19 on people with eye diseases}

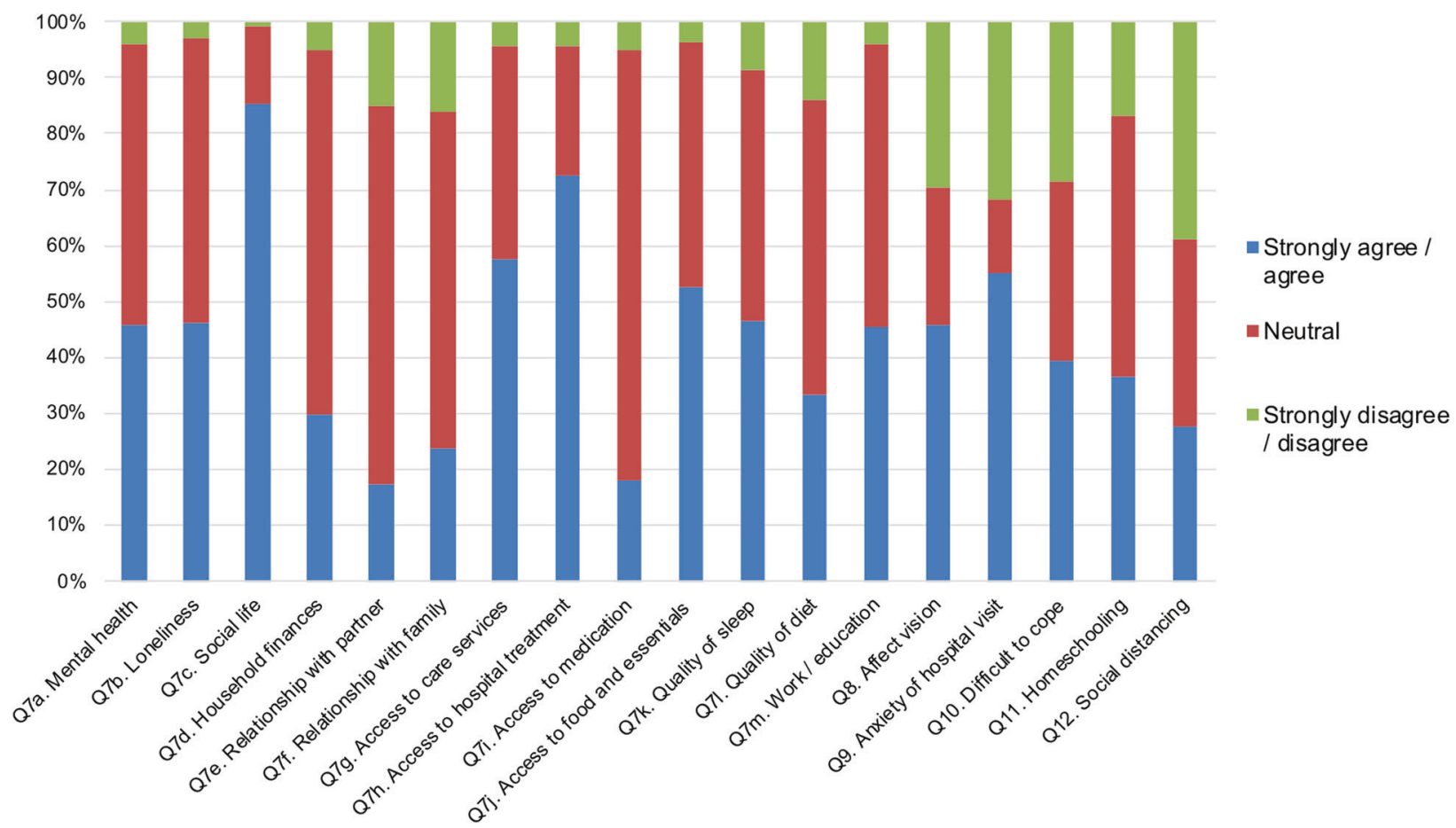

Fig. 1 Psychosocial impact of COVID-19 pandemic lockdown on people living with eye diseases. The questions (Q) asked in each column corresponds with the question number listed in the questionnaire (see Supplementary Table 1). "Strongly agree/agree" means that the respondents agreed that the lockdown had a negative impact on the questioned aspect and vice versa for "strongly disagree/ disagree".
Table 1 Multivariable logistic regression analyses examining the predictive factors for worsened mental health, loneliness, and fear of further sight loss in people living with eye diseases during the COVID-19 pandemic lockdown.

\begin{tabular}{|c|c|c|c|c|c|c|}
\hline & $\begin{array}{l}\text { Mental health } \\
\text { OR }(95 \% \text { CI) }\end{array}$ & $P$ value & $\begin{array}{l}\text { Loneliness } \\
\text { OR }(95 \% \mathrm{CI})\end{array}$ & $P$ value & $\begin{array}{l}\text { Fear of sight loss } \\
\text { OR }(95 \% \text { CI })\end{array}$ & $P$ value \\
\hline \multicolumn{7}{|l|}{ Age, years } \\
\hline $18-64$ & $3.16(1.81-5.52)$ & $\leq 0.001$ & $1.30(0.76-2.23)$ & 0.35 & $1.00(0.50-1.99)$ & $>0.99$ \\
\hline 65 or older & Reference & - & Reference & - & Reference & - \\
\hline \multicolumn{7}{|l|}{ Gender } \\
\hline Male & Reference & - & Reference & - & Reference & - \\
\hline Female & $1.44(0.80-2.59)$ & 0.23 & $1.60(0.88-2.91)$ & 0.12 & $0.91(0.43-1.94)$ & 0.81 \\
\hline \multicolumn{7}{|l|}{ Ethnicity } \\
\hline White & Reference & - & Reference & - & Reference & - \\
\hline BAME & $0.34(0.07-1.61)$ & 0.18 & $0.96(0.23-3.99)$ & 0.96 & $1.03(0.17-6.07)$ & 0.98 \\
\hline \multicolumn{7}{|l|}{ Extent of sight loss } \\
\hline Mild or no VI & Reference & - & Reference & - & Reference & - \\
\hline Moderate VI & $2.61(1.45-4.70)$ & $\underline{0.001}$ & $2.99(1.69-5.32)$ & $\leq 0.001$ & $1.10(0.54-2.26)$ & 0.79 \\
\hline Severe VI or blind & $2.07(1.02-4.19)$ & 0.044 & $3.02(1.51-6.07)$ & 0.002 & $1.28(0.51-3.22)$ & 0.60 \\
\hline
\end{tabular}

Statistically significant values are underlined.

$O R$ odd ratio, $C I$ confidence interval, BAME Black, Asian and minority ethnicity, VI visual impairment.
Funding DSJT acknowledges support from the Medical Research Council/Fight for Sight Clinical Research Fellowship (MR/T001674/ 1) and the Fight for Sight/John Lee, Royal College of Ophthalmologists Primer Fellowship (24CO4).

\section{Compliance with ethical standards}

Conflict of interest The authors declare that they have no conflict of interest. 
Publisher's note Springer Nature remains neutral with regard to jurisdictional claims in published maps and institutional affiliations.

\section{References}

1. McGinty EE, Presskreischer R, Han H, Barry CL. Psychological distress and loneliness reported by US adults in 2018 and April 2020. JAMA. 2020;324:e209740.

2. Brooks SK, Webster RK, Smith LE, Woodland L, Wessely S, Greenberg N, et al. The psychological impact of quarantine and how to reduce it: rapid review of the evidence. Lancet. 2020;395:912-20.
3. Razai MS, Oakeshott P, Kankam H, Galea S, Stokes-Lampard H. Mitigating the psychological effects of social isolation during the covid-19 pandemic. BMJ. 2020;369:m1904.

4. Foot B, MacEwen C. Surveillance of sight loss due to delay in ophthalmic treatment or review: frequency, cause and outcome. Eye. 2017;31:771-5.

5. Ting DSJ, Foo VH, Yang LWY, Sia JT, Ang M, Lin H, et al. Artificial intelligence for anterior segment diseases: emerging applications in ophthalmology. Br J Ophthalmol. 2020. https://doi. org/10.1136/bjophthalmol-2019-315651 